\title{
Efficacy and safety of Huazhi Rougan granule in the treatment of non-alcoholic fatty liver: a systematic review and meta-analysis
}

\author{
Tianyuan Wang ${ }^{1,2}$, Jin Song ${ }^{1,2}$, Jing Hu ${ }^{1,2}$, Shuo Feng ${ }^{1,2}$, Huina Zhang ${ }^{1,2}$, Hong Wang ${ }^{1,2}$, Bo Li ${ }^{1,2}$ \\ ${ }^{1}$ Beijing Hospital of Traditional Chinese Medicine, Capital Medical University, Beijing, China; ${ }^{2}$ Beijing Institute of Traditional Chinese Medicine, \\ Beijing, China \\ Contributions: (I) Conception and design: T Wang, B Li; (II) Administrative support: None; (III) Provision of study materials or patients: T Wang; (IV) \\ Collection and assembly of data: T Wang, J Song, B Li, H Wang, H Zhang; (V) Data analysis and interpretation: S Feng, J Hu, B Li; (VI) Manuscript \\ writing: All authors; (VII) Final approval of manuscript: All authors. \\ Correspondence to: Bo Li. Beijing Hospital of Traditional Chinese Medicine, Capital Medical University, No. 23 Art Gallery Back Street, Dongcheng \\ District, Beijing 100010, China. Email: libo@bjzhongyi.com.
}

Background: This study aims to systematically assess the effectiveness and safety of Huazhi Rougan granule in the treatment of non-alcoholic fatty liver.

Methods: The PubMed, Cochrane Library, EMBASE, China National Knowledge Infrastructure (CNKI), WanFang databases, VIP databases, Chinese Biomedical Literature Database (SinoMed) were searched to identify the relevant randomized controlled trials, from the establishment of the database to June 7 , 2020. The Cochrane risk of bias tool for assessing risk of bias was employed to evaluate the quality of the literatures included. Meta-analyses were performed using RevMan 5.3 software.

Results: A total of 23 related literature were retrieved, 9 studies with 1,142 participants were included in the meta-analysis. The overall quality of evidence for this study is low. Meta-analysis results suggest that, Huazhi Rougan granule combined with conventional treatment was significantly superior to the silybin control group in the improvement of liver B-ultrasound, reduction of serological indexes, increase of highdensity lipoprotein and total clinical effective rate, which was statistically significant. The improvement of serum lipid parameters included alanine aminotransferase (ALT): [mean deviation (MD) $=-10.49,95 \%$ confidence interval (CI): $-17.09,-3.90, \mathrm{P}<0.05]$, aspartate transaminase (AST): $(\mathrm{MD}=-9.44,95 \% \mathrm{CI}$ : -14.62, -4.26, $\mathrm{P}<0.05)$, total cholesterol (TC): (MD =-0.77, 95\% CI: $-0.94,-0.60, \mathrm{P}<0.05)$, triglyceride (TG): $(\mathrm{MD}=-0.40,95 \% \mathrm{CI}:-0.56,-0.24, \mathrm{P}<0.05)$. Reduce low-density lipoprotein cholesterol: $(\mathrm{MD}=-0.40$, 95\% CI: $-0.56,-0.24, \mathrm{P}<0.05)$. Clinical effective rate: [risk ratio $(\mathrm{RR})=1.25,95 \% \mathrm{CI}: 1.16,1.36, \mathrm{P}<0.05$ ]. Occurrence of adverse reactions: Of the 9 studies included, 5 reported adverse reactions, of which 3 reported no drug-related adverse reactions. Adverse reactions were reported in 2 cases, all of which were mild adverse reactions.

Conclusions: The clinical efficacy of Huazhi Rougan granule combined with conventional basic therapy in the treatment of non-alcoholic fatty liver may be better than that of conventional basic therapy combined with silybin, which may improve the B-ultrasonic grading effect of the liver and reduce the serum lipid parameters of the patients. it may improve the clinical symptoms of non-alcoholic fatty liver, and the incidence of adverse reactions is low, but the number of existing clinical studies is small and the quality is low, in order to further verify the above conclusions. More high-quality clinical RCT trials need to be carried out, and internationally recognized outcome indicators should be selected and uniformly included in the scoring criteria.

Keywords: Huazhi Rougan granules; non-alcoholic fatty liver disease (NAFLD); meta-analysis; system evaluation

Submitted Aug 15, 2020. Accepted for publication Dec 22, 2020.

doi: 10.21037/apm-20-1613

View this article at: http://dx.doi.org/10.21037/apm-20-1613 


\section{Introduction}

Non-alcoholic fatty liver disease (NAFLD) is one of the most common chronic liver diseases worldwide, with a gradually increasing number of cases in China (1). NAFLD is a metabolic stress liver injury closely related to insulin resistance (IR) and genetic susceptibility. Its pathological changes are similar to those of alcoholic liver disease (ALD), but the patients have no history of excessive drinking. The disease spectrum includes non-alcoholic hepatic steatosis, nonalcoholic steatohepatitis (NASH) and its associated cirrhosis and hepatocellular carcinoma $(1,2)$.

According to the study, the prevalence rate of NAFLD in ordinary adults is $6.3-45 \%$, the highest in the Middle East and South America, the lowest in Africa, and the uppermiddle level of NAFLD in most countries in Asia, including China (3). Hu et al. (4) reported that the occurrence of NAFLD in China has obvious differences in region, sex, age, occupation and race, which may be related to different living environment, customs and dietary structure, and there may also be differences in genetic background and susceptibility among different races. Compared with the general population, patients with NAFLD had a higher overall mortality, and the hazard ratios was 1.038 . The main causes of death were cardiovascular disease, malignant tumor, liver disease-related death and various infections (5). At the moment the etiopathogenesis of NAFLD is not defined yet and the 'two hit theory' was proposed for the pathogenic mechanism. The main risk factors are obesity, type 2 diabetes, oxidative stress, endoplasmic reticulum stress (ERS), immune response and inflammation, genetic susceptibility, Toll-like receptors, etc. (6).

The existing conventional treatment drugs are mainly weight loss drugs: orlistat and sibutramine; drugs for regulating metabolic disorders: metformin, thiazolidinediones, angiotensin receptor blockers, statins; liver protection and anti-inflammatory drugs: vitamin E, ursodeoxycholic acid, silymarin, Polyene phosphatidylcholine, etc. (7). However, so far, there is still no clear and effective treatment, and the adverse reactions of western medicine are large, and there are many adverse reactions. Traditional Chinese medicine with syndrome differentiation and treatment as the theoretical guidance, the usage of the whole concept of prevention and treatment of this disease has its unique advantages, less side effects, and has made some progress in theoretical and clinical research (8). Therefore, how to make rational use of traditional Chinese medicine to prevent and treat nonalcoholic fatty liver and improve its curative effect needs further research.

Clinical trial results demonstrate that (9-17) Huazhi Rougan granule may effectively improve the B-ultrasonic score of liver in patients with NAFLD and reduce its serum and blood lipid indexes effectively, may improve the clinical symptoms of non-alcoholic fatty liver. But there has been no systematic review or meta-analysis comparing the efficacy and safety of Huazhi Rougan granules in treating non-alcoholic fatty liver. This study aims to systematically evaluate the evidence on the safety and effectiveness of Huazhi Rougan granules in treating non-alcoholic fatty liver from randomized controlled trials.

Therefore, a meta-analysis was conducted in the present study to comprehensively evaluate the efficacy and safety of Huazhi Rougan granule in the treatment of nonalcoholic fatty liver, in order to provide evidence for clinical application.

We present the following article in accordance with the PRISMA reporting checklist (available at http://dx.doi. org/10.21037/apm-20-1613).

\section{Methods}

\section{Retrieval strategy}

The PubMed, Cochrane Library, EMBASE, CNKI, WanFang databases, VIP databases, SinoMed were searched to identify the relevant randomized controlled trials, from the establishment of the database to June 7 , 2020. Search by using the combination of subject words and free words. Retrieval words including (Huazhi Rougan Granules OR Huazhirougan) AND (non-alcoholic fatty liver OR non-alcoholic liver steatosis OR non-alcoholic steatohepatitis OR liver cirrhosis OR hepatocellular carcinoma) AND (randomized clinical trial OR randomized OR RCT). Retrieve completed but unpublished research on ClinicalTrials.gov and follow up the results.

\section{Clinical trial inclusion criteria}

The eligibility criteria are summarized using the PICOS approach (patients, intervention, comparisons, outcomes and study design type). (I) Study design adopted a randomized controlled trial, was conducted regardless of whether the blind method was used. (II) The study subjects were The patients with NADFLD in accordance with the diagnostic criteria of 2018 guidelines for the diagnosis and treatment of non-alcoholic fatty liver disease (2). It is clear that the diagnosis 
of NAFLD must meet the following 3 conditions (18-22): (i) no history of drinking or alcohol consumption equivalent to less than $140 \mathrm{~g} /$ week (women < $70 \mathrm{~g} /$ week); (ii) excluding specific diseases that can cause fatty liver such as viral hepatitis, drug-induced liver disease, total parenteral nutrition, hepatolenticular degeneration, autoimmune liver disease; (iii) the histological changes of liver biopsy meet the pathological diagnostic criteria of fatty liver disease. (III) The experimental group was treated with Huazhirugan granule combined with routine basic treatment (including improving diet, maintaining a good state of mind, proper exercise, etc.), while the control group was treated with routine basic treatment (including improving diet, maintaining a good state of mind, appropriate exercise, etc.) combined with silybin drugs (including silybin capsules, silybin meglumine tablets). Exclusion criteria were: (i) research on incomplete or unable to extract data; (ii) repeatedly published research.

\section{Outcome indicator}

The primary outcome measure was B-ultrasonic grading effect of the liver, while secondary outcome measures included serological index [alanine aminotransferase (ALT), aspartate aminotransferase (AST), gammaglutamyltransferase (GGT)], blood lipid index [total cholesterol (TC), triglyceride (TG), high-density lipoprotein-cholesterol (HDL-C), low-density lipoprotein cholesterol (LDL-C)], clinical effective rate, Clinical symptom score and adverse events.

\section{Literature screening and data extraction}

Two researchers, Tianyuan Wang and Jin Song, independently screened the literature according to the literature selection criteria, and combined with the relevant characteristics of the literature included in this study, the literature information extraction table was developed. it mainly includes the following information: (I) the name of the first author; (II) the publication time; (III) the sample size; (IV) the average age of the subjects; (V) the intervention measures; (VI) the outcome index. In case of disagreement, consult Bo Li, a third-party evaluator, and discuss it with Hong Wang and Huina Zhang.

\section{Assessment of risk of bias}

According to the literature evaluation criteria (23) provided by CochraneHandbook5.3.0, the included studies were evaluated for bias risk, including the generation of random sequences, allocation hiding, blind evaluation of subjects and researchers, blind evaluation of study outcomes, integrity of outcome data, selective reporting of study outcomes and other biases. Other biases include whether there is a conflict of interest in the experiment. If each item is satisfied, it is low risk, and if it is not satisfied, it is high risk. If there is no sufficient information in the study, the bias risk is unknown. In addition, the quality of the included research methodology was evaluated item by item according to the literature evaluation standard (23) provided by CochraneHandbook5.3.0. Bias risk assessment and methodological quality assessment were carried out independently by Shuo Feng and Jing Hu. When there was disagreement, the opinions were unified through consultation with Bo Li.

\section{Data synthesis and statistical analysis}

The statistical analysis of the results of the study was performed by RevMan5.3 software. Dichotomous variables were assessed using relative risk, with $95 \%$ confidence intervals. Continuous variables with the same intervention and measurement method or the unit is exactly the same were analyzed using mean differences and $95 \%$ confidence intervals. Different measurement methods or different units of continuous variables choose standardized mean difference as statistics and $95 \%$ confidence interval. T The heterogeneity $\mathrm{Q}$ statistic test was used to analyze heterogeneity among the included trials (test level $\alpha=0.1$ ) combined with $\mathrm{I}^{2} .0<\mathrm{I}^{2} \leq 50 \%$ is considered to have no obvious heterogeneity, so the fixed effect model is selected. $\mathrm{I}^{2} \geq 50 \%$ is considered to be heterogeneity, and the source of heterogeneity needs to be analyzed. If the clinical heterogeneity is not obvious, random effect model is selected.

\section{Results}

\section{Study selection}

According to the retrieval strategy, 73 related articles were searched, including PubMed $(\mathrm{n}=1)$, Embase $(\mathrm{n}=0)$, Cochrane Library $(n=0)$, CNKI $(n=18)$, SinoMed $(n=17)$, VIP Database $(\mathrm{n}=17)$ and WanFang Database $(\mathrm{n}=19)$. After excluding duplicate articles, there were 23 remaining studies and the remaining 20 articles are selected after reading titles and abstracts are selected. Nine articles are selected 


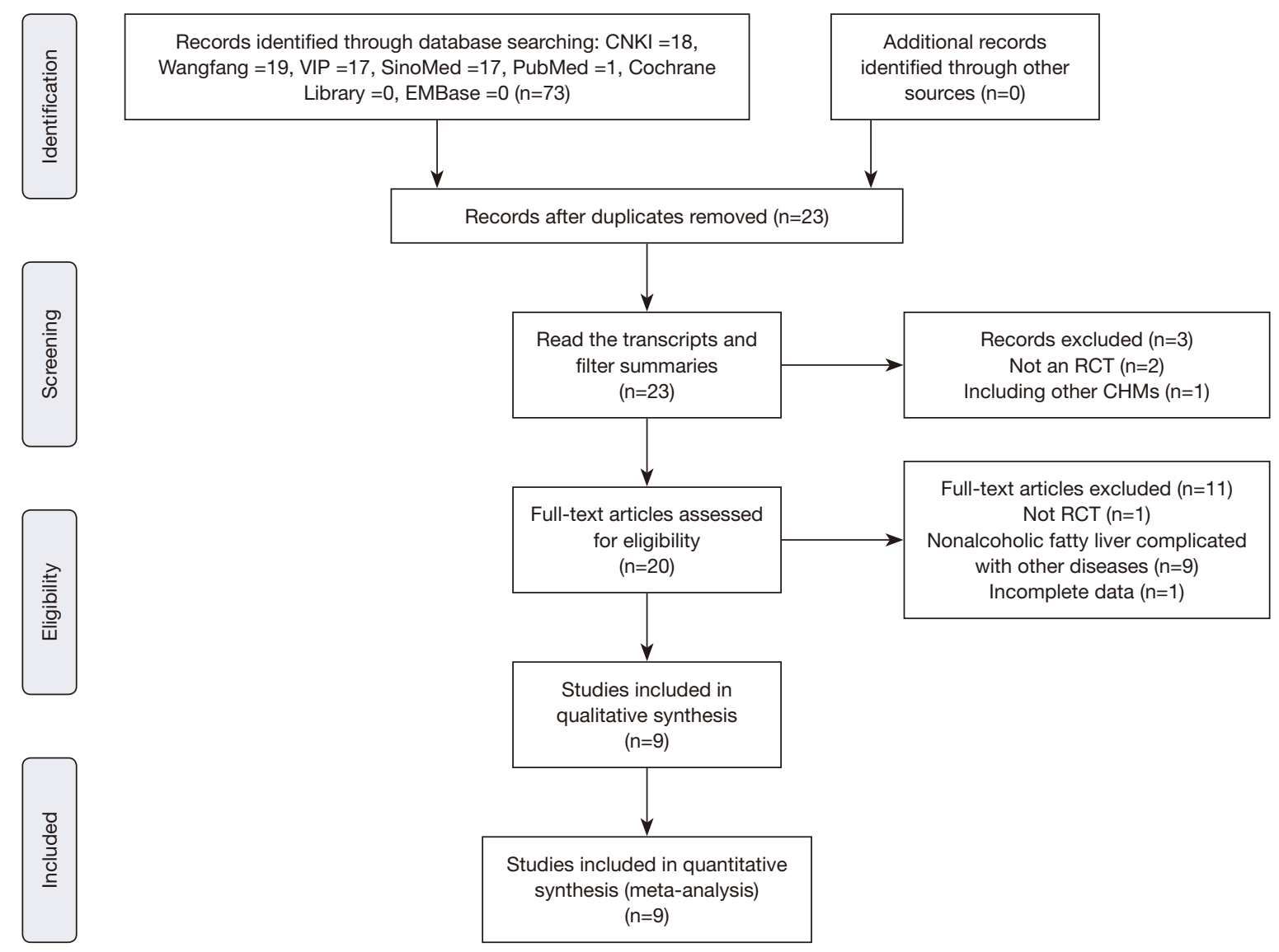

Figure 1 Literature screening flow diagram.

according to the selection criteria of the reading text. The specific literature screening process is shown on flow diagram (Figure 1).

\section{Study characteristics}

A total of 9 articles (9-17) were included in this study, all of which were RCT studies, 1,142 subjects were included. The age range was 18 to 80 . All patients were treated with Huazhi Rougan granule combined with basic treatment and silybin capsule combined with basic treatment. The basic features are summarized in detail (Table 1).

\section{Risk of bias within studies}

The methodological qualities of the included studies were illustrated in Quality evaluation form (Table 2). The overall quality of the included studies was low (Figures 2,3). Randomization was mentioned in all studies, of which 6
$(9-11,13,14,16)$ described the randomized method, of which $4(10,13,14,16)$ were random number table methods, and $2(9,11)$ used the wrong random method-according to the order of admission. The random method is not mentioned in item $3(12,15,17)$. All studies have not designed the allocation hiding method, blind method. None of the studies blinded the researcher subjects. All the studies did not evaluate the outcome of the study by blind method. All studies have integrity for the completed outcome data, but $2(13,16)$ have lost follow-up, reported the number of lost follow-up, but did not explain the reason and the way to deal with it. None of the studies selectively reported the results of the study. No conflict of interest was mentioned in all studies.

\section{Data analysis}

Primary outcome

B-ultrasonic grading effect of the liver: one (16) study 
Table 1 The basic features of the included study

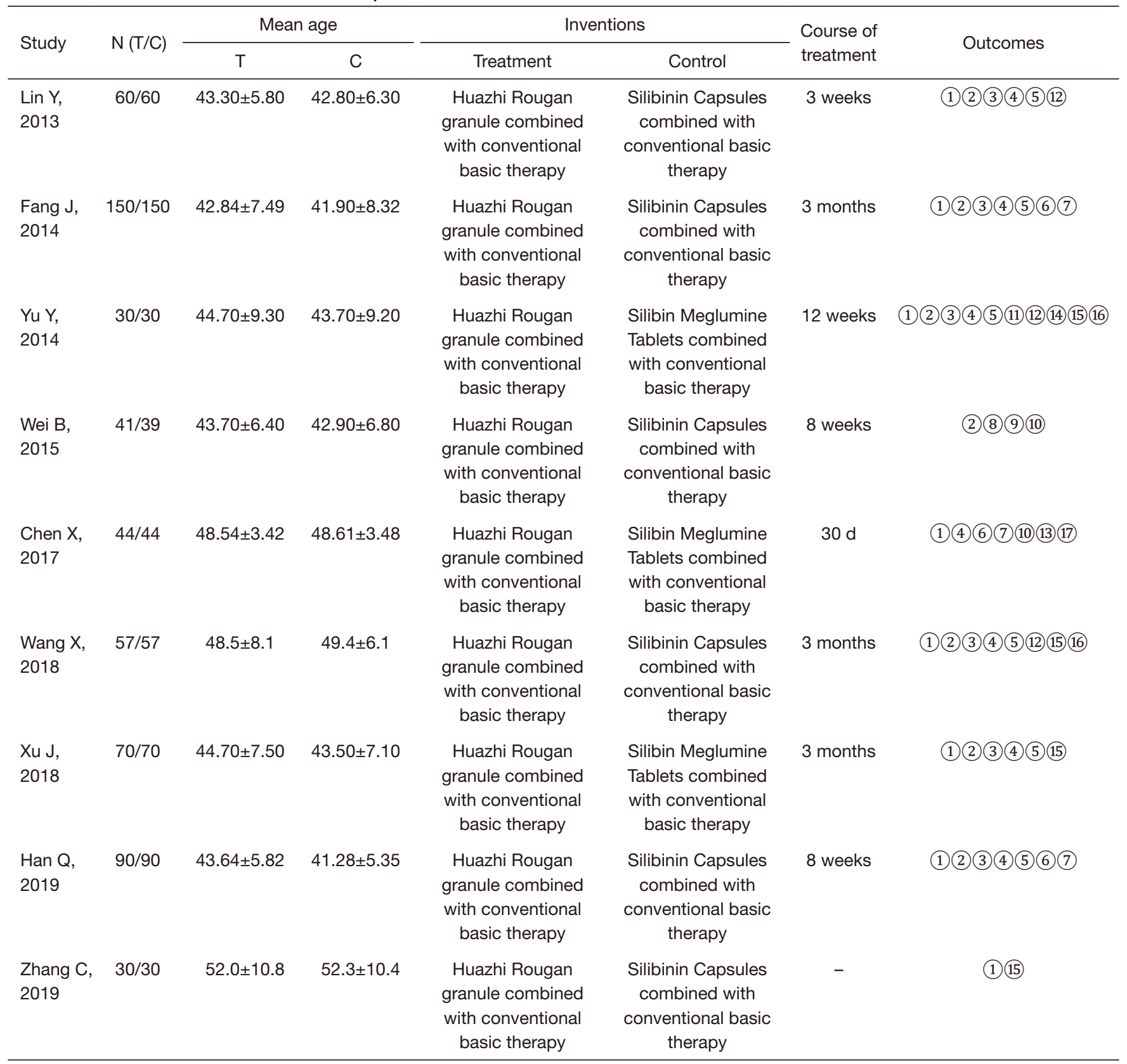

(1): clinical effective rate; (2): ALT; (3): AST; (4): TG; (5): TC; (6): HDL-C; (7): LDL-C; (8): DAO; (9): ET; (10): TNF- $\alpha$; (11): GGT; (12): BMI; (13): ADPN; (14): B-ultrasonic grading effect of the liver; (15): clinical symptom score; 16): adverse effects rate; (17): IL-6.

reported 60 patients. The experimental group was treated with Huazhi Rougan granule combined with routine basic treatment (including improving dietary structure, maintaining a good state of mind, proper exercise, etc.), while the control group was treated with routine basic treatment (including improving dietary structure, maintaining a good state of mind, proper exercise, etc.) combined with silybin meglumine tablets.

The liver B-ultrasound was examined before and after treatment, which was performed by the same physician. Two B-ultrasound doctors examined and recorded the liver morphology, liver surface, intrahepatic parenchyma echo, 
Table 2 Quality evaluation of research methodology

\begin{tabular}{|c|c|c|c|c|c|c|c|}
\hline Study & $\begin{array}{l}\text { Random } \\
\text { sequence } \\
\text { generation }\end{array}$ & $\begin{array}{l}\text { Allocation } \\
\text { concealment }\end{array}$ & $\begin{array}{l}\text { Blinding of } \\
\text { participants and } \\
\text { personnel }\end{array}$ & $\begin{array}{l}\text { Blinding of } \\
\text { outcome } \\
\text { assessment }\end{array}$ & $\begin{array}{l}\text { Incomplete outcome } \\
\text { data }\end{array}$ & $\begin{array}{l}\text { Selective } \\
\text { reporting }\end{array}$ & Other bias \\
\hline $\begin{array}{l}\text { Yu Y, } \\
2014\end{array}$ & $\begin{array}{l}\text { Random number } \\
\text { table }\end{array}$ & $\begin{array}{l}\text { Unallocated } \\
\text { hiding }\end{array}$ & $\begin{array}{l}\text { The researchers } \\
\text { and subjects were } \\
\text { not blinded }\end{array}$ & $\begin{array}{l}\text { No outcome } \\
\text { blind evaluation } \\
\text { was performed }\end{array}$ & $\begin{array}{l}\text { A total of } 3 \text { cases } \\
\text { were lost and followed } \\
\text { up. The reasons and } \\
\text { treatment methods } \\
\text { were not explained }\end{array}$ & $\begin{array}{l}\text { Results were } \\
\text { not selectively } \\
\text { reported }\end{array}$ & $\begin{array}{l}\text { Not } \\
\text { mentioned }\end{array}$ \\
\hline $\begin{array}{l}\text { Chen X, } \\
2017\end{array}$ & $\begin{array}{l}\text { According to } \\
\text { the order of } \\
\text { admission }\end{array}$ & $\begin{array}{l}\text { Unallocated } \\
\text { hiding }\end{array}$ & $\begin{array}{l}\text { The researchers } \\
\text { and subjects were } \\
\text { not blinded }\end{array}$ & $\begin{array}{l}\text { No outcome } \\
\text { blind evaluation } \\
\text { was performed }\end{array}$ & $\begin{array}{l}\text { Complete outcome } \\
\text { data }\end{array}$ & $\begin{array}{l}\text { Results were } \\
\text { not selectively } \\
\text { reported }\end{array}$ & $\begin{array}{l}\text { Not } \\
\text { mentioned }\end{array}$ \\
\hline $\begin{array}{l}\text { Wang X, } \\
2018\end{array}$ & $\begin{array}{l}\text { Random number } \\
\text { table }\end{array}$ & $\begin{array}{l}\text { Unallocated } \\
\text { hiding }\end{array}$ & $\begin{array}{l}\text { The researchers } \\
\text { and subjects were } \\
\text { not blinded }\end{array}$ & $\begin{array}{l}\text { No outcome } \\
\text { blind evaluation } \\
\text { was performed }\end{array}$ & $\begin{array}{l}\text { A total of } 6 \text { cases } \\
\text { were lost and followed } \\
\text { up. The reasons and } \\
\text { treatment methods } \\
\text { were not explained }\end{array}$ & $\begin{array}{l}\text { Results were } \\
\text { not selectively } \\
\text { reported }\end{array}$ & $\begin{array}{l}\text { Not } \\
\text { mentioned }\end{array}$ \\
\hline $\begin{array}{l}\text { Xu J, } \\
2018\end{array}$ & Not mentioned & $\begin{array}{l}\text { Unallocated } \\
\text { hiding }\end{array}$ & $\begin{array}{l}\text { The researchers } \\
\text { and subjects were } \\
\text { not blinded }\end{array}$ & $\begin{array}{l}\text { No outcome } \\
\text { blind evaluation } \\
\text { was performed }\end{array}$ & $\begin{array}{l}\text { Complete outcome } \\
\text { data }\end{array}$ & $\begin{array}{l}\text { Results were } \\
\text { not selectively } \\
\text { reported }\end{array}$ & $\begin{array}{l}\text { Not } \\
\text { mentioned }\end{array}$ \\
\hline
\end{tabular}

intrahepatic vascular course and intrahepatic color blood flow signal.

After treatment, the B-ultrasonic grading of liver in the experimental group changed from "severe fatty liver" ( $n=4)$, "moderate fatty liver" ( $n=8)$, "mild fatty liver" $(n=18)$ and normal $(\mathrm{n}=0)$. After treatment, it became "severe fatty liver" in 0 cases, "moderate fatty liver" in 2 cases, "mild fatty liver" in 11 cases and normal in 17 cases. The B-ultrasonic grading of liver in the experimental group changed from "severe fatty liver" ( $n=4)$, "moderate fatty liver" ( $n=8)$, "mild fatty liver" ( $n=18)$ and normal $(n=0)$. After treatment, there were 0 cases of "severe fatty liver", 2 cases of "moderate fatty liver", 11 cases of "mild fatty liver" and 17 cases of normal liver $(\mathrm{P}<0.05)$. The $\mathrm{B}$-ultrasonic grading of liver in the control group changed from "severe fatty liver" $(n=3)$, "moderate fatty liver" ( $\mathrm{n}=10)$, "mild fatty liver" $(\mathrm{n}=17)$ and normal ( $\mathrm{n}=0)$. After treatment, it became "severe fatty liver" in 1 case, "moderate fatty liver" in 5 cases, "mild fatty liver" in 10 cases and normal in 14 cases $(\mathrm{P}<0.05)$ (Table 3).

\section{Secondary outcomes}

$A L T$

A total of 814 patients were included in $6(10,12-16)$ studies. A total of 408 patients received routine basic treatment 


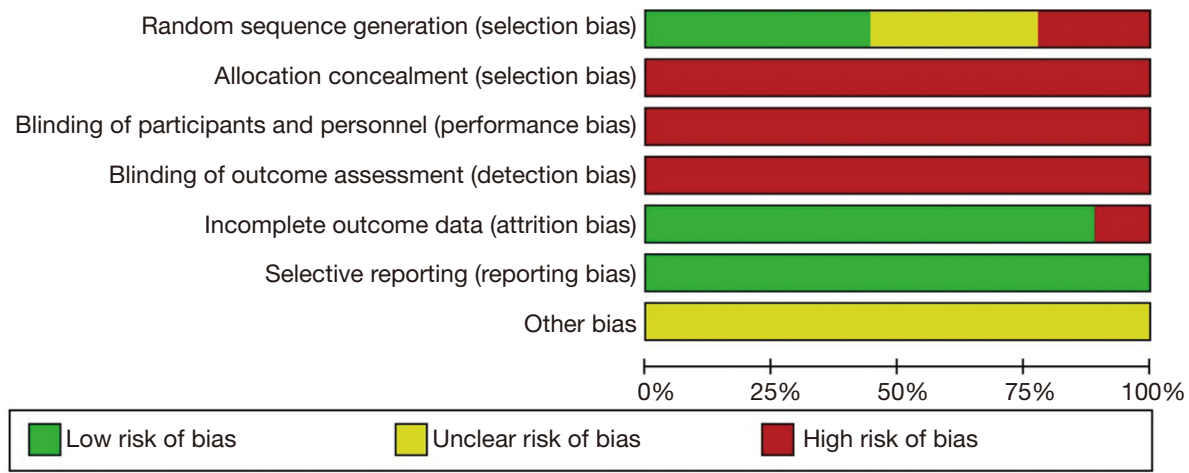

Figure 2 Risk of bias graph.

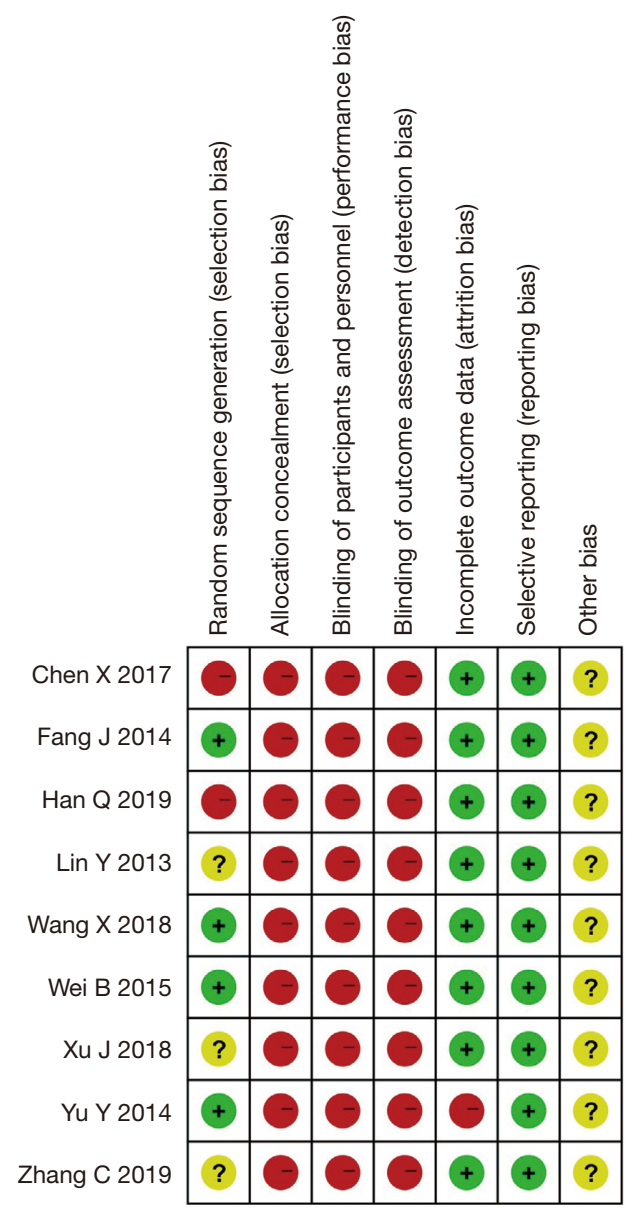

Figure 3 Risk of bias summary.

(including improving diet, maintaining a good state of mind, proper exercise, etc.). combined with Huazhi Rougan granule, a total of 406 patients were treated with routine basic treatment (including improving dietary structure, maintaining a good state of mind, proper exercise, etc.) and silibinin meglumine tablets, including silybin capsules and silibinin meglumine tablets. Heterogeneity test showed that there was significant heterogeneity between groups $\left(\mathrm{P}<0.05, \mathrm{I}^{2}=90 \%\right)$. Results showed that the effect of Huazhi Rougan granule combined with routine basic treatment (including improving dietary structure, maintaining a good state of mind, proper exercise, etc.) was better than that of the control group which was received basic treatment combined with silybin-containing drugs (MD $=-10.49$, 95\% CI: $-17.09,-3.90, \mathrm{P}<0.05)$, the difference was statistically significant (Figure 4).

$A S T$

A total of 734 patients were included in $5(10,12-15)$ studies. A total of 367 patients in the experiment group were treated with routine basic treatment (including improving diet, maintaining a good state of mind, proper exercise, etc.). combined with Huazhi Rougan granule, a total of 367 patients were treated with routine basic treatment (including improving diet, maintaining a good state of mind, proper exercise, etc.) and silibinin meglumine tablets (including silybin capsules and silibinin meglumine tablets). Heterogeneity test showed that there was significant heterogeneity between groups $\left(\mathrm{P}<0.05, \mathrm{I}^{2}=86 \%\right)$. The results showed that the effect of histochemical Huazhi Rougan granule combined with routine basic treatment (including improving dietary structure, maintaining a good state of mind, proper exercise, etc.) was better than that of the control group in reducing AST (including improving dietary structure and maintaining a good state of mind, proper exercise, etc.). combined with silybin drugs (MD $=-9.44,95 \%$ CI: $-14.62,-4.26, \mathrm{P}<0.05)$, the difference was 
Table $3 \mathrm{Yu}$ Y studied the results of liver B-ultrasonic grading

\begin{tabular}{lcccccc}
\hline Group & $\mathrm{N}$ & Times & Severe fatty liver & Moderate fatty liver & Light fatty live & Normality \\
\hline Treatment & 30 & Prior treatment & 4 & 8 & 18 & 1 \\
& & Posttreatment & 0 & 2 & 11 & 1 \\
Control & 30 & Prior treatment & 3 & 5 & 17 & 17 \\
& & Posttreatment & 1 & 5 & 14 \\
\hline
\end{tabular}

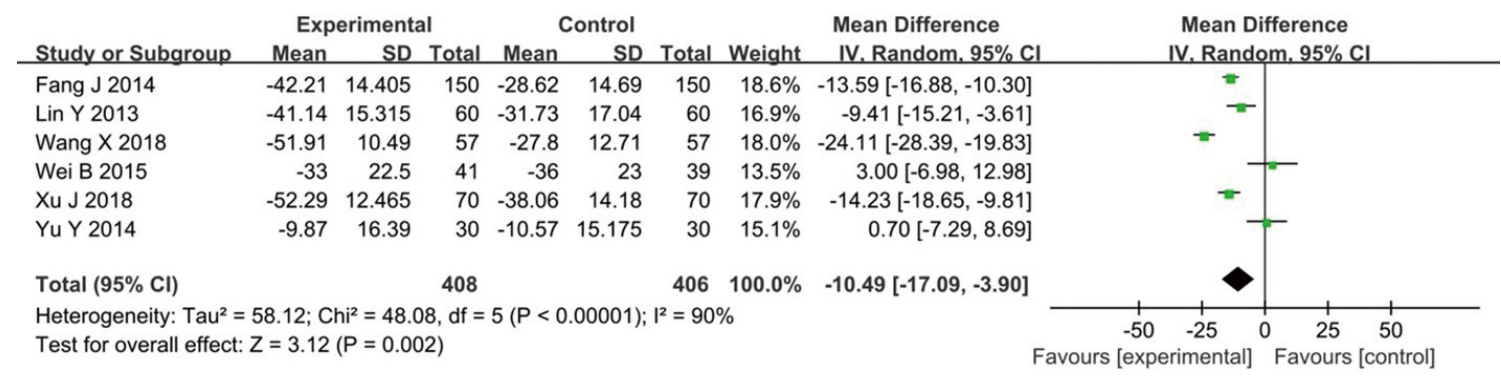

Figure 4 Forest plots of ALT. ALT, alanine aminotransferase.

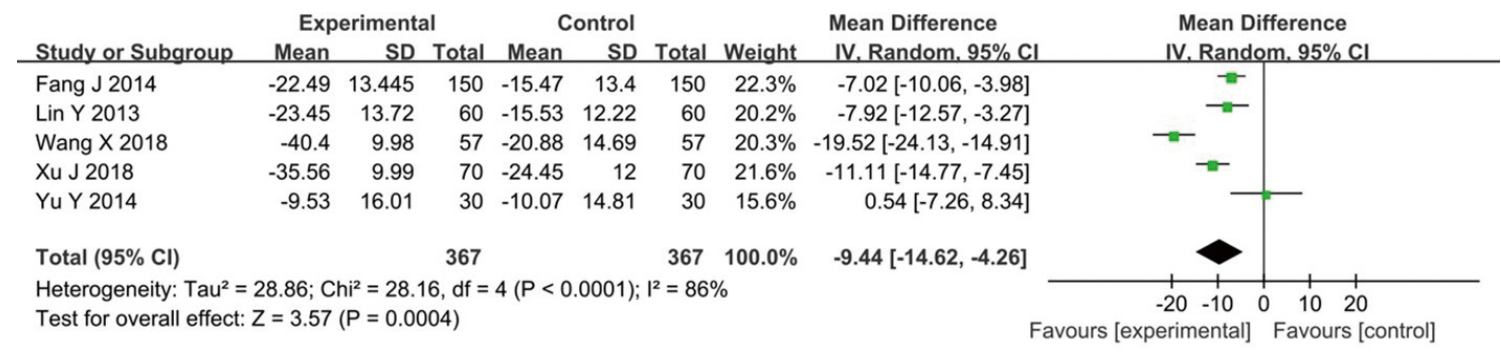

Figure 5 Forest plots of AST. AST, aspartate transaminase.

statistically significant (Figure 5).

\section{GGT}

A total of 60 patients were included in 1 (16) study. Thirty patients in the control group were treated with routine basic treatment (including improving diet, maintaining a good state of mind, proper exercise, etc.). combined with Huazhi Rougan granule, a total of 30 patients in the control group were treated with routine basic treatment (including improving dietary structure, maintaining a good state of mind, proper exercise, etc.) combined with silibinin meglumine tablets, including silybin capsules and silibinin meglumine tablets. The level of TGG, test group decreased from $47.60 \pm 24.33$ to $36.13 \pm 13.24$ IU/L $(\mathrm{P}<0.05)$ before and after treatment, and the difference was statistically significant. In the control group, it decreased from $52.27 \pm 26.74$ to $37.93 \pm 12.16$ IU/L $(\mathrm{P}<0.05)$, and the difference was statistically significant.

\section{$T C$}

A total of 734 patients were included in $6(9,10,12,13,15,16)$ studies. A total of 367 patients were treated with Huazhi Rougan granule combined with basic treatment (including improving diet, maintaining a good state of mind, proper exercise, etc.). The control group received routine basic treatment (including improving diet, maintaining a good state of mind, proper exercise, etc.) combined with silybin drugs (including silybin capsules, silibinin meglumine tablets), a total of 367 patients. There was a small heterogeneity among studies $\left(\mathrm{P}=0.70, \mathrm{I}^{2}=0 \%\right)$. The results showed that the effect of histochemical Huazhi Rougan granule combined with routine basic treatment (including improving dietary structure, maintaining a good state of mind, proper exercise, etc.) on reducing TC was better 


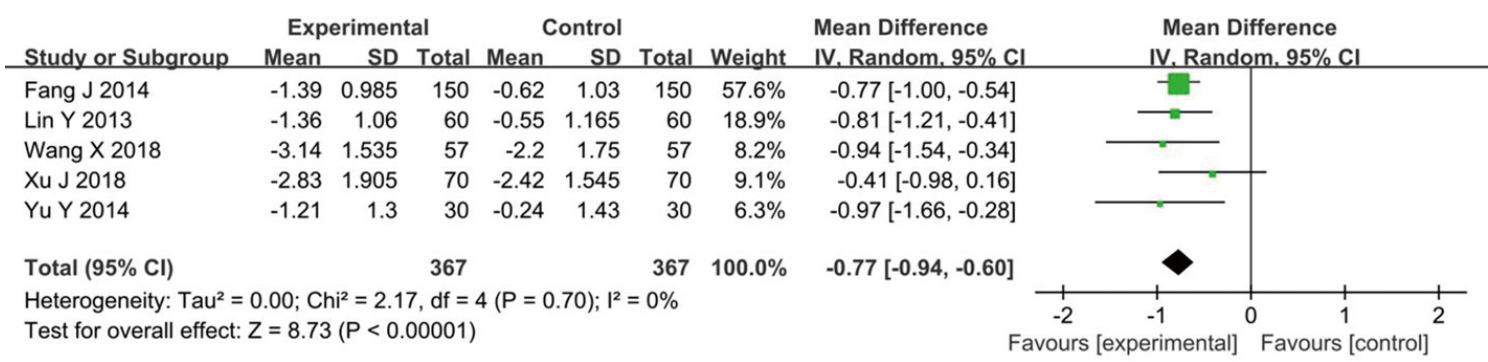

Figure 6 Forest plots of TC. TC, total cholesterol.

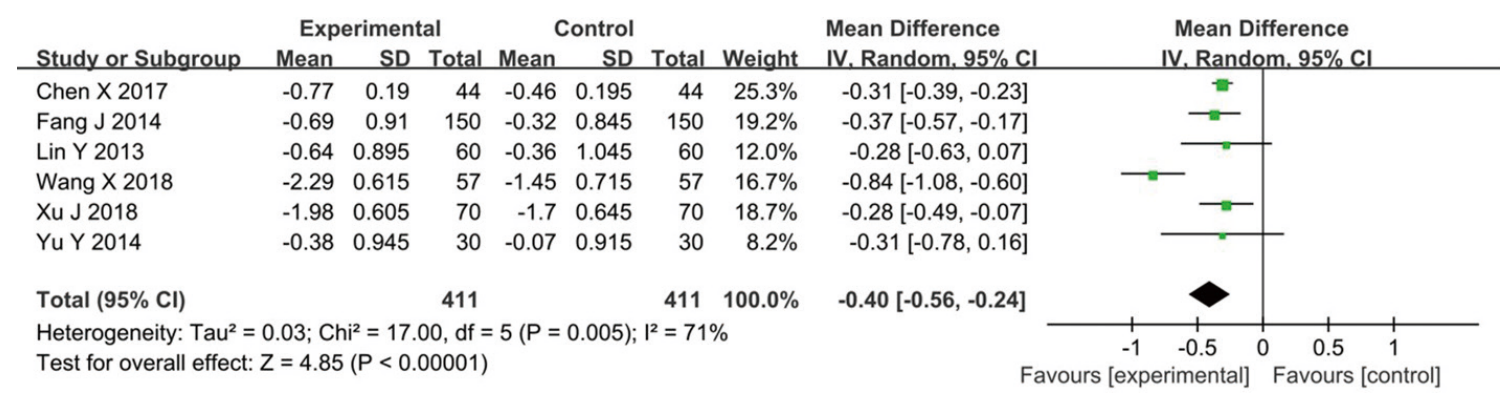

Figure 7 Forest plots of TG. TG, triglyceride.

than that in the control group (including improving dietary structure, maintaining a good state of mind, proper exercise, etc.) combined with silybin drugs ( $\mathrm{MD}=-0.77,95 \% \mathrm{CI}$ : $-0.94,-0.60, \mathrm{P}<0.05)$, the difference was statistically significant (Figure 6).

TG

A total of 822 patients were included in $6(9,10,12,13,15,16)$ studies. A total of 411 patients were treated with Huazhi Rougan granule combined with basic treatment (including improving diet, maintaining a good state of mind, proper exercise, etc.). The control group received routine basic treatment (including improving diet, maintaining a good state of mind, proper exercise, etc.) combined with silybin drugs (including silybin capsules, silibinin meglumine tablets), a total of 411 people. Heterogeneity test showed that there was significant heterogeneity between groups $\left(\mathrm{P}=0.005, \mathrm{I}^{2}=71 \%\right)$. The results showed that the effect of histochemical Huazhi Rougan granule combined with routine basic treatment (including improving dietary structure, maintaining a good state of mind, proper exercise, etc.) was better than that of the control group in reducing TG (including improving dietary structure and maintaining a good state of mind, appropriate exercise, etc.) combined with silybin-containing drugs $(\mathrm{MD}=-0.40,95 \% \mathrm{CI}:-0.56$, $-0.24, \mathrm{P}<0.05)$, the difference was statistically significant
(Figure 7).

\section{$H D L-C$}

A total of 388 patients were included in $2(9,10)$ studies. Experimental histochemical Huazhi Rougan granule combined with basic treatment (including improving diet, maintaining a good state of mind, proper exercise, etc.) The control group was treated with routine basic treatment (including improving diet, maintaining a good state of mind, proper exercise, etc.) combined with silybin drugs (including silybin capsules, silibinin meglumine tablets). Heterogeneity test showed that there was significant heterogeneity between groups. The results showed that the effect of histochemical Huazhi Rougan granule combined with routine basic treatment (including improving dietary structure, maintaining a good state of mind, proper exercise, etc.) was better than that of the control group (including improving dietary structure and maintaining a good state of mind.) the effect of increasing HDL-C was better than that of the control group (including improving dietary structure and maintaining a good state of mind, Appropriate exercise, etc.) combined with silybin drugs $(\mathrm{SMD}=0.38$, 95\% CI: $-0.33,1.09, \mathrm{P}<0.05)$, the difference was statistically significant (Figure 8).

\section{$L D L-C$}

A total of 388 patients were included in $2(9,10)$ studies. 


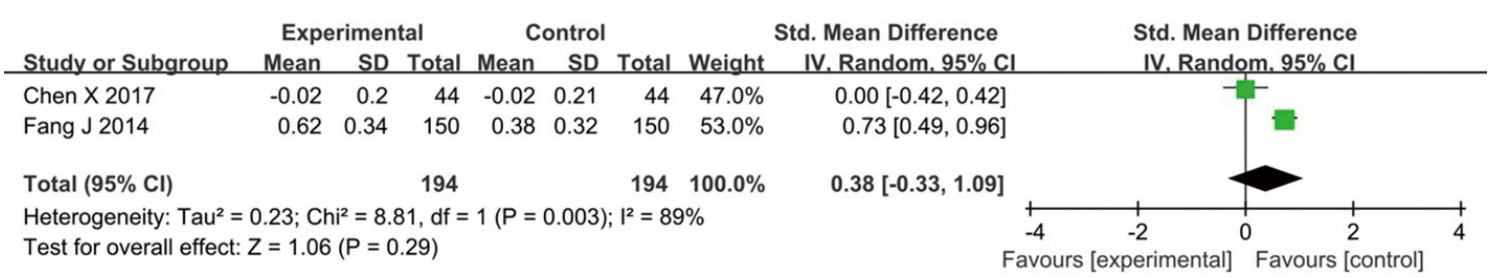

Figure 8 Forest plots of HDL-C. HDL-C, high-density lipoprotein cholesterol.

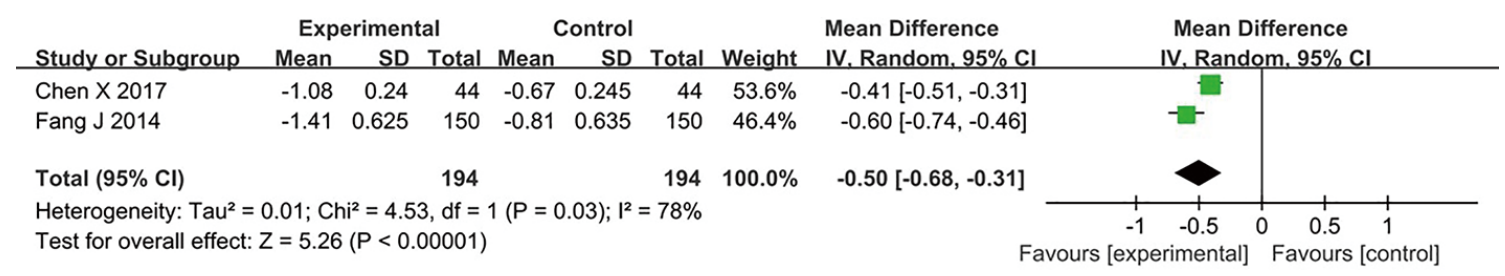

Figure 9 Forest plots of LDL-C. LDL-C, low-density lipoprotein cholesterol.

Trial histochemical combination of Huazhi Rougan granule and basic treatment (including improving dietary structure, maintaining a good state of mind, proper exercise, etc.), a total of 194 people. The control group was treated with routine basic treatment (including improving diet, maintaining a good state of mind, proper exercise, etc.) combined with silybin drugs (including silybin capsules, silibinin meglumine tablets), a total of 194 people. Heterogeneity test showed that there was significant heterogeneity between groups $\left(\mathrm{P}=0.03, \mathrm{I}^{2}=78 \%\right)$. The results showed that the effect of histochemical Huazhi Rougan granule combined with routine basic treatment (including improving dietary structure, maintaining a good state of mind, proper exercise, etc.) was better than that of the control group in reducing LDL-C (including improving dietary structure and maintaining a good state of mind, appropriate exercise, etc.) combined with silybin-containing drugs $(M D=-0.50,95 \% \mathrm{CI}:-0.68,-0.31, \mathrm{P}<0.05)$, the difference was statistically significant (Figure 9).

\section{Clinical effective rate}

A total of 1,062 patients were included in 8 (9-13,15-17) studies. A total of 531 patients were treated with Huazhi Rougan granule combined with routine basic treatment (including improving dietary structure, maintaining a good state of mind, proper exercise, etc.), the number of effective people accounted for $72.9 \%$ of the total number of people in the test group. The control group received routine basic treatment (including improving diet, maintaining a good state of mind, proper exercise, etc.) combined with silybin drugs (including silybin capsules, silibinin meglumine tablets), a total of 531 patients, the effective number accounted for $58.2 \%$ of the total number of people in the test group. There was a small heterogeneity among studies $\left(\mathrm{P}=0.83, \mathrm{I}^{2}=0 \%\right)$ and the fixed effect model was used. The results showed that the clinical effective rate of experimental histochemical Huazhi Rougan granule combined with routine basic treatment (including improving dietary structure, maintaining a good state of mind, proper exercise, etc.) was significantly higher than that of the control group (including improving dietary structure, maintaining a good state of mind, proper exercise, etc.) combined with silybin group ( $\mathrm{RR}=1.25,95 \% \mathrm{CI}: 1.16,1.36, \mathrm{P}<0.05)$, The difference is statistically significant (Figure 10).

\section{Clinical symptom score and adverse events}

Four $(11,13,16,17)$ studies reported clinical symptoms and signs. The experimental group was treated with Huazhi Rougan granule combined with routine basic treatment (including improving diet, maintaining a good state of mind, proper exercise, etc.). The control group was treated with routine basic treatment (including improving diet, maintaining a good state of mind, proper exercise, etc.) combined with drugs containing silybin (including silybin capsules, silibinin meglumine tablets). The study takes liver pain, fatigue, sticky stool, poor appetite, dry mouth and bitter mouth as indicators, using different evaluation methods, so there is no data merging. The results showed that both the experimental group and the control group could improve the clinical symptoms of non-alcoholic fatty liver after treatment, and the improvement of the experimental group was better than that of the control 


\begin{tabular}{|c|c|c|c|c|c|}
\hline \multirow[b]{2}{*}{ Study or Subgroup } & \multicolumn{2}{|c|}{ Experimental } & \multicolumn{2}{|c|}{ Control } & \multirow[b]{2}{*}{ Weight } \\
\hline & Events & Total & Events & Total & \\
\hline Chen X 2017 & 40 & 44 & 31 & 44 & $10.0 \%$ \\
\hline Fang J 2014 & 78 & 150 & 64 & 150 & $20.7 \%$ \\
\hline Han Q 2019 & 85 & 90 & 71 & 90 & $23.0 \%$ \\
\hline Lin Y 2013 & 56 & 60 & 48 & 60 & $15.5 \%$ \\
\hline Wang X 2018 & 51 & 57 & 38 & 57 & $12.3 \%$ \\
\hline Xu J 2018 & 34 & 70 & 27 & 70 & $8.7 \%$ \\
\hline Yu Y 2014 & 14 & 30 & 7 & 30 & $2.3 \%$ \\
\hline Zhang C 2019 & 29 & 30 & 23 & 30 & $7.4 \%$ \\
\hline Total $(95 \% \mathrm{CI})$ & & 531 & & 531 & $100.0 \%$ \\
\hline Total events & 387 & & 309 & & \\
\hline \multicolumn{6}{|c|}{ Heterogeneity: $\mathrm{Chi}^{2}=3.56, \mathrm{df}=7(P=0.83) ;\left.\right|^{2}=0 \%$} \\
\hline
\end{tabular}

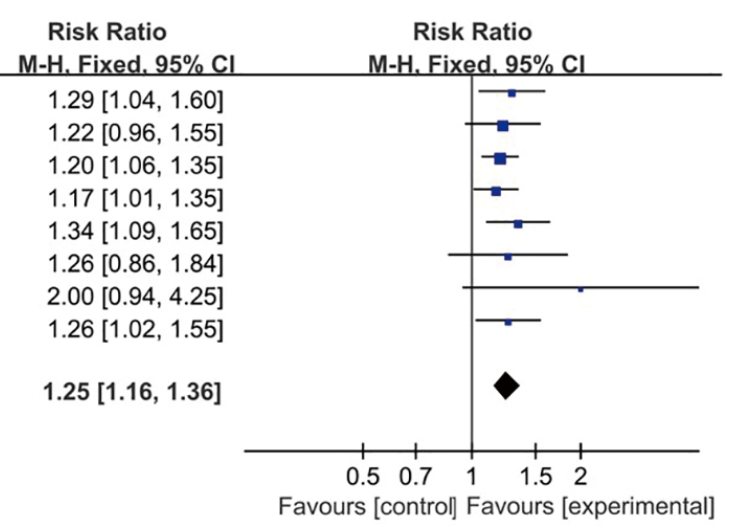

Figure 10 Forest plots of clinical effective rate.

group $(\mathrm{P}<0.05)$.

\section{Adverse reaction}

Of the 9 (9-17) studies included, 5 reported adverse reactions, including 12 cases of drug taste discomfort, 1 case of diarrhea and no other obvious adverse reactions in the observation group during the treatment cycle. There were 4 cases of nausea and gastrointestinal reactions and 3 cases of skin rash in the control group. Yu Yang reported that there were 1 case of epigastric discomfort and 1 case of mild diarrhea in the treatment group, 1 case of mild abdominal distension and 1 case of mild dizziness in the control group. All of them were not given special treatment and relieved spontaneously within 1 week. Fang et al., Han et al., and Wei et al. all reported no drug-related adverse reactions.

\section{Evaluation of GRADE evidence quality}

The software GRADEprofiler3.6 was used to grade the evidence of each outcome index. The factors that affect the quality of evidence are described in detail in Figure 11.

\section{Discussion}

\section{The main findings of this study}

With the rapid development of the economy and the change of life style, NAFLD is one of the most important public health problem worldwide and NAFLD closely associated with obesity, cardiovascular and cerebrovascular diseases, hypertension, dyslipidemia and other diseases (24). It seriously affects the life quality of patients. Nonalcoholic fatty liver disease total mortality is higher than others ordinary disease.

Huazhi Rougan granules are composed of Artemisiae Scopariae, cassia seed (stir-fried), rhubarb (wine stew), alisma alisma, Polyporus umbellatus, Hawthorn,
Atractylodes macrocephala (fried with bran), Atractylodes macrocephala (fried with bran), tangerine peel, Trichosanthes, Ligustrum lucidum (steamed with wine), Chinese wolfberry, thistle, Bupleurum (fried with vinegar). Huazhi Rougan granules can treat nonalcoholic simple fatty liver. Symptoms include abdominal discomfort and abdominal pain or dull pain, fatigue, anorexia.

Pharmacological studies have shown that Artemisiae Scopariae has a significant effect on hepatoprotective effects. The mechanism of hepatoprotective effects is complicated. It includes preventing hepatocyte necrosis, protecting hepatocyte membrane integrity and good permeability, enhancing liver detoxification function, promoting hepatocyte regeneration and improving liver microcirculation (25). Cassia seed extract contains polysaccharides, glycosides, proteins and anthraquinones, which can significantly reduce the contents of serum triglyceride and low density lipoprotein cholesterol and increase the level of serum high density lipoprotein cholesterol in hyperlipidemic animals (26). Aloe-emodin in rhubarb can protect mice from acute liver injury induced by CC intestine, prevent the death of hepatocytes, promote the synthesis of glutamine synthetase and albumin in liver, and detoxify by combining ammonia with glutamate to form glutamine (27). Alisma alisma has the effect of lowering blood sugar and blood pressure, in which choline, lecithin and other components have certain anti-fatty liver effect (28). Polyporus umbellatus polysaccharides can inhibit hepatocyte injury induced by $\mathrm{CCl} 4$, reduce the activities of glutamic oxaloacetic transaminase (AST), glutamic pyruvic transaminase (ALT) and malondialdehyde (MDA) in hepatocytes, increase the survival rate of hepatocytes, and significantly induce the expression of CYP3AmRNA, which 


\begin{tabular}{|c|c|c|c|c|c|c|}
\hline \multicolumn{7}{|c|}{$\begin{array}{l}\text { Clinical symptom response rate for non-alcoholic Fatty liver } \\
\text { Patient or population: patients with non-alcoholic Fatty liver } \\
\text { Settings: } \\
\text { Intervention: Clinical symptom response rate }\end{array}$} \\
\hline Outcomes & $\begin{array}{l}\text { Illu } \\
\text { Assumed risk } \\
\text { Control }\end{array}$ & $\begin{array}{l}\text { parative risks }{ }^{\star}(95 \% \mathrm{Cl}) \\
\text { Corresponding risk } \\
\text { Clinical symptom response rate }\end{array}$ & $\begin{array}{l}\text { Relative effect } \\
\quad(95 \% \mathrm{Cl})\end{array}$ & $\begin{array}{l}\text { No of Participants } \\
\text { (studies) }\end{array}$ & $\begin{array}{l}\text { Quality of the } \\
\text { evidence } \\
\text { (GRADE) }\end{array}$ & Comments \\
\hline $\begin{array}{l}\text { Clinical symptom } \\
\text { response rate }\end{array}$ & $\begin{array}{l}582 \text { per } 1000 \\
686 \text { per } 1000\end{array}$ & $\begin{array}{l}\text { dy population } \\
\quad 727 \text { per } 1000 \text { ( } 675 \text { to } 791) \\
\text { Moderate } \\
\quad 858 \text { per } 1000 \text { (796 to } 933)\end{array}$ & $\begin{array}{c}\text { RR } 1.25 \\
\text { (1.16 to } 1.36)\end{array}$ & $\begin{array}{c}1062 \\
\text { (8 studies) }\end{array}$ & $\begin{array}{c}\oplus \ominus \ominus \ominus \\
\text { very low }\end{array}$ & \\
\hline
\end{tabular}

${ }^{\star}$ The basis for the assumed risk (e.g. the median control group risk across studies) is provided in footnotes. The corresponding risk (and its $95 \%$ confidence interval) is based on the assumed risk in the comparison group and the relative effect of the intervention (and its $95 \% \mathrm{Cl}$ ).

Cl: Confidence interval; RR: Risk ratio:

GRADE Working Group grades of evidence

High quality: Further research is very unlikely to change our confidence in the estimate of effect.

Moderate quality: Further research is likely to have an important impact on our confidence in the estimate of effect and may change the estimate.

Low quality: Further research is very likely to have an important impact on our confidence in the estimate of effect and is likely to change the estimate.

Very low quality: We are very uncertain about the estimate.

${ }^{1}$ There is a risk of bias: 2 items used the wrong random method, 3 items did not mention the random method, none designed the allocation hidden method or blind method, neither

blinded the study subjects, neither blindly evaluated the study outcome, nor explained the reasons for the reported loss of follow-up and the way to deal with it.

${ }^{2}$ Uncalculated sample size

${ }^{3}$ There is publication bias.

Serological indicator for non-alcoholic Fatty liver

Patient or population: patients with non-alcoholic Fatty liver

Settings:

Intervention: Serological indicator

\begin{tabular}{|c|c|c|c|c|c|c|}
\hline \multirow[b]{2}{*}{ Outcomes } & \multicolumn{2}{|c|}{ Illustrative comparative risks ${ }^{\star}(95 \% \mathrm{Cl})$} & \multirow[b]{2}{*}{$\begin{array}{l}\text { Relative effect } \\
\qquad(95 \% \mathrm{Cl})\end{array}$} & \multirow[b]{2}{*}{$\begin{array}{l}\text { No of Participants } \\
\text { (studies) }\end{array}$} & \multirow{2}{*}{$\begin{array}{l}\text { Quality of the } \\
\text { evidence } \\
\text { (GRADE) }\end{array}$} & \multirow[b]{2}{*}{ Comments } \\
\hline & $\begin{array}{l}\text { Assumed risk } \\
\text { Control }\end{array}$ & $\begin{array}{c}\text { Corresponding risk } \\
\text { Serological indicator }\end{array}$ & & & & \\
\hline ALT & & $\begin{array}{c}\text { The mean alt in the intervention groups was } \\
0.75 \text { standard deviations lower } \\
\text { (1.28 to } 0.21 \text { lower) }\end{array}$ & & 814 (6 studies) & $\begin{array}{c}\oplus \ominus \ominus \ominus \\
\text { very low }\end{array}$ & $\begin{array}{c}\text { SMD }-0.75 \\
(-1.28 \text { to }-0.21)\end{array}$ \\
\hline AST & & $\begin{array}{c}\text { The mean ast in the intervention groups was } \\
9.44 \text { lower (14.62 to } 4.26 \text { lower) }\end{array}$ & & 734 (5 studies) & $\begin{array}{c}\oplus \ominus \ominus \ominus \\
\text { very low } \\
\end{array}$ & \\
\hline
\end{tabular}

${ }^{*}$ The basis for the assumed risk (e.g. the median control group risk across studies) is provided in footnotes. The corresponding risk (and its $95 \%$ confidence interval) is based on the assumed risk in the comparison group and the relative effect of the intervention (and its $95 \% \mathrm{Cl}$ ).

Cl: Confidence interval;

GRADE Working Group grades of evidence

High quality: Further research is very unlikely to change our confidence in the estimate of effect.

Moderate quality: Further research is likely to have an important impact on our confidence in the estimate of effect and may change the estimate.

Low quality: Further research is very likely to have an important impact on our confidence in the estimate of effect and is likely to change the estimate.

Very low quality: We are very uncertain about the estimate.

1 There is a risk of bias: two random methods are not mentioned; no allocation hidden method or blind method is designed; neither of them is blind to the study subjects; both are blind evaluation of the study outcome; the reasons for the reported loss of follow-up and the way to deal with it are not explained.

${ }^{2}$ Heterogeneity test showed that there was great heterogeneity among groups. $\mathrm{P}<0.00001, \mathrm{I}^{2}=90$

${ }^{3}$ Uncalculated sample size

${ }^{4}$ Heterogeneity test showed that there was great heterogeneity among groups. $P<0.00001, I^{2}=86$

Lipid index for non-alcoholic Fatty liver

Patient or population: patients with non-alcoholic Fatty liver

Settings:

Intervention: Lipid index

\begin{tabular}{|c|c|c|c|c|c|c|}
\hline \multirow[b]{2}{*}{ Outcomes } & \multicolumn{2}{|c|}{ Illustrative comparative risks ${ }^{\star}(95 \% \mathrm{Cl})$} & \multirow[b]{2}{*}{$\begin{array}{l}\text { Relative effect } \\
(95 \% \mathrm{Cl})\end{array}$} & \multirow[b]{2}{*}{$\begin{array}{l}\text { No of Participants } \\
\text { (studies) }\end{array}$} & \multirow{2}{*}{$\begin{array}{l}\text { Quality of the } \\
\text { evidence } \\
\text { (GRADE) }\end{array}$} & \multirow[b]{2}{*}{ Comments } \\
\hline & $\begin{array}{l}\text { Assumed risk } \\
\text { Control }\end{array}$ & $\begin{array}{c}\text { Corresponding risk } \\
\text { Lipid index }\end{array}$ & & & & \\
\hline LDL-C & & $\begin{array}{c}\text { The mean Idl-c in the intervention groups was } \\
0.5 \text { lower ( } 0.68 \text { to } 0.31 \text { lower) }\end{array}$ & & 388 (2 studies) & $\begin{array}{c}\oplus \ominus \ominus \ominus \\
\text { very low }\end{array}$ & \\
\hline HDL-C & & $\begin{array}{c}\text { The mean hdl-c in the intervention groups was } \\
0.38 \text { standard deviations higher } \\
\text { ( } 0.33 \text { lower to } 1.09 \text { higher) }\end{array}$ & & 388 (2 studies) & $\begin{array}{c}\oplus \oplus \ominus \ominus \\
\text { very low }\end{array}$ & $\begin{array}{c}\text { SMD } 0.38 \\
(-0.33 \text { to } 1.09)\end{array}$ \\
\hline $\mathrm{TG}$ & & $\begin{array}{c}\text { The mean } \mathrm{tg} \text { in the intervention groups was } \\
0.4 \text { lower ( } 0.56 \text { to } 0.24 \text { lower) }\end{array}$ & & 822 (6 studies) & $\begin{array}{c}\oplus \oplus \ominus \ominus \ominus \\
\text { very low }\end{array}$ & \\
\hline $\mathrm{TC}$ & & $\begin{array}{c}\text { The mean tc in the intervention groups was } \\
0.77 \text { lower ( } 0.94 \text { to } 0.6 \text { lower) }\end{array}$ & & 734 (5 studies) & $\begin{array}{c}\oplus \ominus \ominus \ominus \\
\text { very low }\end{array}$ & \\
\hline
\end{tabular}

*The basis for the assumed risk (e.g. the median control group risk across studies) is provided in footnotes. The corresponding risk (and its $95 \%$ confidence interval) is based on the assumed risk in the comparison group and the relative effect of the intervention (and its $95 \% \mathrm{Cl}$ ).

Cl: Confidence interval;

GRADE Working Group grades of evidence

High quality: Further research is very unlikely to change our confidence in the estimate of effect.

Moderate quality: Further research is likely to have an important impact on our confidence in the estimate of effect and may change the estimate.

Low quality: Further research is very likely to have an important impact on our confidence in the estimate of effect and is likely to change the estimate.

Very low quality: We are very uncertain about the estimate.

${ }^{1}$ There is a risk of bias: one item uses the wrong random method; no allocation hidden method or blind method is designed; none of the study subjects are blind; all of them are blind evaluation of the outcome of the study.

${ }^{2}$ Heterogeneity test showed that there was great heterogeneity among groups. $P=0.03, I^{2}=78$

${ }^{3}$ Uncalculated sample size

${ }^{4}$ Heterogeneity test showed that there was great heterogeneity among groups. $P=0.003, I^{2}=89$

${ }^{5}$ Confidence interval crosses clinical decision threshold

${ }^{6}$ There is a risk of bias: 2 items did not mention the random method, 1 item used the wrong random method; no allocation hidden method or blind method was designed; the study subjects were not blinded; all of them were blind evaluation of the study outcome.

${ }^{7}$ Heterogeneity test showed that there was great heterogeneity among groups. $P=0.005, I^{2}=71$

${ }^{8}$ There is a risk of bias: two random methods are not mentioned; no allocation hidden method or blind method is designed; none of the study subjects are blind; all of them are blind evaluation of the study outcome.

Figure 11 Evidence table of Huazhi Rougan granule in treatment of viral hepatitis. 
can protect the hepatocytes of Jian carp (29). Atractylodes macrocephala Koidz polysaccharide has obvious effect on the prevention and treatment of non-alcoholic steatohepatitis, and has a significant lipid-lowering effect, and has an obvious effect on improving the index of liver injury (30). Volatile oil and methyl hesperidin in tangerine peel also have the effect of dilating blood vessels, increasing coronary flow, lowering blood pressure and slowing down heart rate. Hesperidin also has the effect of lowering cholesterol (31). Under the combination of multiple mechanisms, Ligustrum lucidum has significant effects on chemical, immune, ischemia-reperfusion liver injury and liver fibrosis, and can also prevent liver injury (32). The total flavonoids of thistle can reduce the levels of blood glucose, cholesterol, triglyceride and low density lipoprotein, thus improving the disorder of blood glucose and lipid metabolism (33). Hawthorn and Trichosanthes have the effect of lowering blood pressure and blood lipid $(34,35)$. Atractylodes and Bupleurum have the effect of protecting liver $(36,37)$.

The results of statistical analysis showed that there was statistical difference in the outcome such as AST, ALT, GGT $(\mathrm{P}<0.05)$. These outcomes also have significance in clinical diagnosis (38). Liver enzymes including serum ALT, AST, and GGT were significantly Increased in patients with NAFLD $(39,40)$. In particular, ALT has been widely used as a surrogate marker of NAFLD because it is specific for liver injury and fat accumulation $(41,42)$. For example, the statistical analysis of ALT in the outcome shows that MD $=-10.49$. It means that the ALT of patient's can be reduced by 10.49 . Therefore, it has certain clinical significance. This study obeys a stringent inclusion criteria. A total of 40 RCTs were included. The results of meta analysis show that: (I) The clinical efficacy of Huazhi Rougan granule combined with routine basic therapy in the treatment of non-alcoholic fatty liver may be better than that of conventional basic therapy. (II) Compared with conventional basic therapy combined with silybin, conventional basic therapy combined with Huazhi Rougan granule may effectively improve the efficacy of liver B ultrasonic grading in patients. (III) Compared with conventional basic therapy combined with silybin, conventional basic therapy combined with Huazhi Rougan granule may effectively reduce serum lipid indexes in patients. (IV) Compared with conventional basic therapy combined with silybin, conventional basic therapy combined with Huazhi Rougan granule may improve the clinical symptoms of non-alcoholic fatty liver. (V) There were few adverse reactions in the included studies, and all of them were mild adverse reactions.

\section{Heterogeneity analysis}

The results showed that there was large heterogeneity among the results of alanine aminotransferase ALT $\left(\mathrm{I}^{2}=90 \%\right)$ and aspartate aminotransferase AST $\left(\mathrm{I}^{2}=86 \%\right)$. There is obvious heterogeneity among the results of triacylglycerol TG $\left(I^{2}=71 \%\right)$ and total cholesterol TC $\left(I^{2}=71 \%\right)$.

There was significant heterogeneity among the results of low density lipoprotein LDL-C $\left(\mathrm{I}^{2}=78 \%\right)$. We considered that heterogeneity comes from the severity of the inclusion of these patients disease was inconsistent. In addition, there are differences in age and ethnic groups by using the combination of serology, blood lipids and other indexes, such as fatty liver index, liver steatosis index and so on. The treatment courses selected in the included studies were not uniform and varied a lot, they were 4 weeks, 8 weeks and 12 weeks. It increased clinical heterogeneity to some extent.

\section{Limitations}

\section{Research object}

All the patients included in this study met the diagnostic criteria of non-alcoholic fatty liver, and there was no significant difference in the age range of the patients. However, the severity of the included patients is not uniform, which may affect the results of the analysis.

\section{Intervention}

There was no significant difference in the types of intervention between the experimental group and the control group. The experimental group was treated with Huazhi Rougan granule combined with routine basic treatment (including improving dietary structure, maintaining a good state of mind, proper exercise, etc.). The control group was treated with routine basic treatment (including improving dietary structure and maintaining a good state of mind). Appropriate exercise, etc.) combined with silybin drugs (including silybin capsules, silibinin meglumine tablets). The dosage forms of silybin in the control group were different, but had little effect on the analysis results.

The quality of the 9 (9-17) studies included in this study was low. In terms of random schemes, only $6(9-11,13,14,16)$ described the randomized method, of which $2(9,11)$ used the wrong random method-according to the order of admission. The random method is not mentioned in item 3 
$(12,15,17)$. Nine studies $(9-17)$ are not satisfied in terms of concealment of allocation schemes, blindness of researchers and subjects, and blind evaluation of research outcomes. In terms of the integrity of the outcome data, all studies have integrity for the completed outcome data, but two $(13,16)$ studies reported the loss of follow-up, only the number of loss of follow-up, but did not explain the reason and the way of treatment. There may be biased effects.

\section{Outcome measures}

The analysis results of the outcome indicators selected for inclusion in the study showed high heterogeneity. There are great individual differences in the combination of serum and blood lipid indexes and there were great individual differences in the combination of serum and blood lipid indexes, as well as differences in age and ethnic groups. In the selection of outcome indicators, there are few internationally recognized outcome indicators such as liver imaging efficacy. In addition, the liver imaging efficacy index should not only count the total effective rate, but also increase the statistics of liver B-ultrasonic grading grade, and the transformation number between each grade should also be counted.

In the study, the symptom selection criteria for symptom improvement were different, and the scoring criteria were also different. Two studies $(12,13)$ selected liver pain, fatigue, sticky stool and poor anorexia. Among them, Zhang Chunming's scoring standard is the transformed score. The scoring standard of Wang Xiling is the number of specific cases of clinical symptoms. In 2 studies $(15,16)$, the right side pain, general fatigue, epigastric pain, dry mouth and bitter mouth were selected. Among them, Yu Yang's scoring standard is the transformed score. Xu Junlin score standard is the specific number of clinical symptoms. These may have an impact on the results of the analysis.

Of the 9 studies included, 5 reported adverse reactions, including 1 case of diarrhea and 12 cases of drug taste discomfort in the experimental group during the treatment cycle. In the control group, there were 3 cases of skin rash and 4 cases of nausea and gastrointestinal reaction. Yu Yang reported that there were 1 case of epigastric discomfort and 1 case of mild diarrhea in the test group, 1 case of mild dizziness and 1 case of mild abdominal distension in the control group. Most of the adverse reactions were mild, which were not treated specially and could be relieved by themselves. No drug-related adverse reactions were reported in the other three studies.

\section{Implications for future research}

It is suggested that a large sample RCT test should be carried out in the future. Researchers should refer to Cochrane risk bias assessment tools to design the test scheme more rigorously, strictly ensure the quality of implementation, and improve the quality of research methodology. The clinical heterogeneity should be taken into account in the study, and it is suggested to unify the criteria for the severity of the disease. It is suggested that liver imaging indexes such as liver B-ultrasound grading efficacy should be selected as the main curative effect index, and the first objective index should be the outcome index and a more unified evaluation standard should be established. Attention should also be paid to the statistics of the incidence of adverse reactions so as to make the research results more complete and reliable.

\section{Conclusions}

The clinical efficacy of Huazhi Rougan granule combined with conventional basic therapy in the treatment of nonalcoholic fatty liver may be better than that of conventional basic therapy combined with silybin, which may improve the B-ultrasonic grading effect of the liver and reduce the serum lipid index of the patients. it may improve the clinical symptoms of non-alcoholic fatty liver, and the incidence of adverse reactions is low, and it has good efficacy and safety. It is suggested that clinicians should consider the combination of Huazhi Rougan granule on the basis of routine treatment in the treatment of non-alcoholic fatty liver. Most of the clinical TCM types are damp-heat stasis type, and most of the studies included in this paper are nonalcoholic fatty liver with damp-heat stasis. It is suggested that clinicians should first choose Huazhi Rougan granule when treating damp-heat stasis type of non-alcoholic fatty liver. at the same time, it is also suitable for other types of non-alcoholic fatty liver. However, the number of existing clinical studies is small, and the quality is low. It is suggested that the internationally recognized outcome indicators should be selected and a unified scoring standard should be used to further verify the above conclusions.

\section{Acknowledgments}

Funding: This work was supported by the National Natural Science Foundation of China (81774146) and the "13th Five-Year" National Science and Technology Major Project 
for New Drugs (2019ZX09734001).

\section{Footnote}

Provenance and Peer Review: This article was commissioned by the editorial office, Annals of Palliative Medicine for the series "Narrative \& Evidence-based Medicine for Traditional Medicine: from basic research to clinical practice and trial". The article has undergone external peer review.

Reporting Checklist: The authors have completed the PRISMA reporting checklist. Available at http://dx.doi. org/10.21037/apm-20-1613

Conflicts of Interest: All authors have completed the ICMJE uniform disclosure form (available at http://dx.doi. org/10.21037/apm-20-1613). The series "Narrative \& Evidence-based Medicine for Traditional Medicine: from basic research to clinical practice and trial" was commissioned by the editorial office without any funding or sponsorship. BL served as the unpaid Guest Editor of the series. The authors have no other conflicts of interest to declare.

Ethical Statement: The authors are accountable for all aspects of the work in ensuring that questions related to the accuracy or integrity of any part of the work are appropriately investigated and resolved.

Open Access Statement: This is an Open Access article distributed in accordance with the Creative Commons Attribution-NonCommercial-NoDerivs 4.0 International License (CC BY-NC-ND 4.0), which permits the noncommercial replication and distribution of the article with the strict proviso that no changes or edits are made and the original work is properly cited (including links to both the formal publication through the relevant DOI and the license). See: https://creativecommons.org/licenses/by-nc-nd/4.0/.

\section{References}

1. Fatty liver and alcoholic liver Disease Group, Hepatology Branch of Chinese Medical Association. Guidelines for diagnosis and treatment of nonalcoholic fatty liver disease. Chinese Journal of liver Diseases (electronic version) 2010;2:43-8.

2. National Workshop on Fatty Liver and Alcoholic Liver
Disease, Chinese Society of Hepatology, Chinese Medical Association, Guidelines of prevention and treatment for nonalcoholic fatty liver disease (2018 update). Infect Dis Info 2018;31:393-402+20.

3. Younossi ZM, Koenig AB, Abdelatif D, et al. Global epidemiology of nonalcoholic fatty liver disease-Metaanalytic assessment of prevalence, incidence, and outcomes. Hepatology 2016;64:73-84.

4. Hu Z, Zhang J. Current status of research on nonalcoholic fatty liver disease in China. J Clin Hepatol 2016;32:552-6.

5. Liu C, Li J. Research on Nonalcoholic Fatty Liver Disease. J Jinzhou Medical University 2018;39:108-12.

6. Chi Z. Pathogenesis of non-alcoholic fatty liver disease. World Journal of Chinese digestion 2017;25:670-83.

7. Zhao J. Current status of drug treatment of non-alcoholic fatty liver. Chinese Community Doctor (Medical Specialty) 2013;15:10-1.

8. Ma D. Research Progress of traditional Chinese Medicine in the treatment of non-alcoholic Fatty liver. Cap Med 2013;20:21-3.

9. Chen X, Fan L, Zhang Y, et al. Curative efficacy of Huazhi Rougan granule in treatment of nonalcoholic fatty liver and its influence on levels of adiponectin, IL-6 and TNF-a. Chinese Journal of Biochemical Pharmaceutics 2017;37:65-7.

10. Fang J, Teng X, Pang J, et al. Comparison of efficacy between Huazhi Rougan Granule and Silybin Capsule in the treatment of nonalcoholic fatty liver disease. Chinese Journal of General Practice 2014;12:655-6+8.

11. Han Q. To compare the clinical effect of Silybin capsule and Huazhi Rougan Granule in the treatment of nonalcoholic fatty liver disease. World Latest Medicine Information (Electronic Version) 2019;19:155-6.

12. Lin $\mathrm{Y}, \mathrm{Xu} F, \mathrm{Wu} \mathrm{D}$, et al. Clinical research of Huazhi Rougan Granule in the treatment of non -alcoholic fatty liver disease. Journal of Clinical Medi cine in Pract ice 2013;17:75-7.

13. Wang X, Zhao L, Wang X. Curative Effect Evaluation of Huazhi Rougan Granule on Nonalcoholic Fatty Liver Disease with Damp Heat Accumulation Syndrome. World Chinese Medicine 2018;13:1669-72.

14. Wei B, Chen X, Luo D, et al. Effect of Huizhouruogan Granule on intestinal barrier function in patients with nonalcoholic fatty liver disease. Modern Journal of Integrated Traditional Chinese and Western Medicine 2015;24:2007-9.

15. Xu J, Tao Y. Observe on curative effect of Huazhi Rougan granule in treating Nonalcoholic Fatty liver Disease with damp-heat accumulation. Journal of Clinical Medical 2018;5:79-81. 
16. Yu Y, Qian L, Hou P, et al. Observation of Huazhi Rougan granule in the treatment of nonalcoholic fatty liver disease. Modern Journal of Integrated Traditional Chinese and Western Medicine 2014;23:2302-4.

17. Zhang C. Effect of Dehysteresis and Ruohe Granules on the Treatment of Humid and Hot Deflated Non-alcoholic Liver and its Effect on Clinical Symptoms. Electronic Journal of Clinical Medical Literature 2019;6:20-1.

18. American Gastroenterological Association medical position statement: nonalcoholic fatty liver disease. Gastroenterology 2002;123:1702-4.

19. Fan JG, Farrell GC. Epidemiology of non-alcoholic fatty liver disease in China. J Hepatol 2009;50:204-10.

20. Farrell GC, Chitturi S, Lau GK, et al. Guidelines for the assessment and management of non-alcoholic fatty liver disease in the Asia-Pacific region: executive summary. J Gastroenterol Hepatol 2007;22:775-7.

21. Vuppalanchi R, Chalasani N. Nonalcoholic fatty liver disease and nonalcoholic steatohepatitis: Selected practical issues in their evaluation and management. Hepatology 2009;49:306-17.

22. Zeng MD, Fan JG, Lu LG, et al. Guidelines for the diagnosis and treatment of nonalcoholic fatty liver diseases. J Dig Dis 2008;9:108-12.

23. Savović J, Weeks L, Sterne JA, et al. Evaluation of the Cochrane Collaboration's tool for assessing the risk of bias in randomized trials: focus groups, online survey, proposed recommendations and their implementation. Syst Rev 2014;3:37.

24. He S, Kong J, Niu J. Research status and progress of nonalcoholic fatty liver disease. Chinese Journal of Gerontology 2008:725-7.

25. Liu Y, Qiu X, Liu Y, et al. Research progress on pharmacological effect of Artemisiae Scopariae Herba. Chinese Traditional and Herbal Drugs 2019;50:2235-41.

26. Yang B, Ren J, Qin K, et al. Research progress on pharmacological action and mechanism of Cassia seed. Journal of Chinese Medicinal Materials 2018;41:1247-51.

27. Zhu T, Liu X, Wang X, et al. Progress in research of the changes in pharmacological actions and chemical composition of rhubarbs processed by different methods. Chinese Journal of New Drugs 2016;25:883-7.

28. Xing Z, Chen W, Zeng Y. Research Progress on Chemical Constituents and Pharmacological Effects of Zexie (Alisma orientalis). GUIDING JOURNAL OF TCM 2017;23:75-8.

29. Wang T, Zhang F, Ren Y, et al. Research progress on chemical constituents and pharmacological actions of Polyporus Umbellatus. J Tradit Chin Med 2017;51:109-12.

30. Zhang X, Zuo D. New progress in the study of chemical constituents and pharmacological action of Atractylodes. Information on Traditional Chinese Medicine 2018;35:101-6.

31. Li W. Pharmacological analysis and clinical application of Orange peel. Medical Theory And Practice 2018;31:1521-2+55.

32. Liu X, Liang J, Sun J, et al. Research Progress of the Fructus ligustri lucidi on the Chemical Compounds and Pharmacological Activity. Strait Pharmaceutical Journal 2018;30:1-8.

33. Han W, Dong Y, Sun Y, et al. Pharmacological Action and Clinical Application of Cirsium setosum. Strait Pharmaceutical Journal 2019;31:84-7.

34. Feng R, Zhu X, Zhang M. Research Progress on Pharmacological Function of Hawthorn in The Past Five Years. Journal of Basic Chinese Medicine 2019;25:715-8.

35. Wan L, Lu J, Xu J, et al. Research progress of chemical components and pharmacological action of Trichosanthes kirilowii. China Pharmacy 2015;26:4440-3.

36. Wang X, Zhao Y, Wang X, et al. Research progress on chemical constituents and pharmacological actions of vinegar-baked Radix Bupleuri. Drug Evaluation Research 2018;41:163-8.

37. Zhang M, Shen Y. Research Advances on Difference of Pharmacologic Effects of Atractylodis Rhizoma before and after Processing. Anti Infect Pharm 2017;14:481-5.

38. Chen Z, Han CK, Pan LL, et al. Serum alanine aminotransferase independently correlates with intrahepatic triglyceride contents in obese subjects. Dig Dis Sci 2014;59:2470-6.

39. Omagari K, Kadokawa Y, Masuda J, et al. Fatty liver in non-alcoholic non-overweight Japanese adults: incidence and clinical characteristics. J Gastroenterol Hepatol 2002;17:1098-105.

40. Wong CA, Araneta MR, Barrett-Connor E, et al. Probable NAFLD, by ALT levels, and diabetes among FilipinoAmerican women. Diabetes Res Clin Pract 2008;79:133-40.

41. Scheig R. Evaluation of tests used to screen patients with liver disorders. Prim Care 1996;23:551-60.

42. Yamada J, Tomiyama H, Yambe M, et al. Elevated serum levels of alanine aminotransferase and gamma glutamyltransferase are markers of inflammation and oxidative stress independent of the metabolic syndrome. Atherosclerosis 2006;189:198-205.

Cite this article as: Wang T, Song J, Hu J, Feng S, Zhang H, Wang H, Li B. Efficacy and safety of Huazhi Rougan granule in the treatment of non-alcoholic fatty liver: a systematic review and meta-analysis. Ann Palliat Med 2021;10(12):12969-12984. doi: 10.21037/apm-20-1613 